\section{Case Reports in Oncology}

\title{
A Rare Case of Synchronous Waldenström's Macroglobulinemia and Cutaneous Squamous Cell Carcinoma with a Lung Mass: A Diagnostic and Management Dilemma
}

\author{
Catherine Lynne Limberis ${ }^{a}$ Simon Nayler ${ }^{b, c} \quad$ Bernardo Leon Rapoport ${ }^{d, e}$ \\ aDepartment of Internal Medicine, Bedford Gardens Hospital, Johannesburg, South Africa; \\ bDrs Gritzman and Thatcher Inc. Laboratories, Randburg, Johannesburg, South Africa; \\ 'Wits Donald Gordon Medical Centre, Parktown, Johannesburg, South Africa; ${ }^{\text {dThe Medical }}$ \\ Oncology Centre of Rosebank, Saxonwold, Johannesburg, South Africa; ${ }^{\text {eDepartment of }}$ \\ Immunology, Faculty of Health Sciences, University of Pretoria, Pretoria, South Africa
}

Keywords

Waldenström's macroglobulinemia - Cutaneous squamous cell carcinoma - Metastatic cutaneous squamous cell carcinoma - Squamous cell carcinoma of the lung - Synchronous cancer

\begin{abstract}
Cutaneous squamous cell carcinoma has presented an increasing burden globally, with the occurrence of metastatic cutaneous squamous cell carcinoma being a relatively rare event but presenting with significant challenges in management, and a paucity of treatment options. Waldenström's macroglobulinemia is similarly an infrequent diagnosis. We present a rare case of a synchronous diagnosis of cutaneous squamous cell carcinoma and Waldenström's macroglobulinemia with an associated lung mass with squamous differentiation. The considered origin of the lung mass was either metastatic cutaneous squamous cell carcinoma or a primary squamous cell carcinoma of the lung, representing a third primary malignancy. The report highlights complexities in diagnosis and management, particularly in a patient with multiple synchronous malignancies. It further emphasizes the need for expanded global availability of specific therapies, including PD-1 inhibitors.




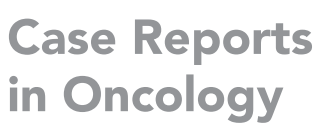

\begin{tabular}{l|l}
\hline Case Rep Oncol 2020;13:1474-1482 \\
\hline DOI: 10.1159/000511460 & $\begin{array}{l}\text { @ 2020 The Author(s). Published by S. Karger AG, Basel } \\
\text { www.karger.com/cro }\end{array}$ \\
\hline
\end{tabular}

Limberis et al.: A Case of Synchronous Waldenström's Macroglobulinemia and cSCC with a Lung Mass

\section{Introduction}

Cutaneous squamous cell carcinoma (CSCC) and other non-melanoma skin cancers have posed a progressively greater challenge for global health systems, with the occurrence of metastatic cSCC being a relatively rare event [1]. Independently, Waldenström's macroglobulinemia is, likewise, an uncommon disorder. While there is data to support associated malignancies in the setting of a Waldenström's macroglobulinemia, most of the data describe preexisting or subsequent malignancies, including non-melanoma skin cancers and lung cancer $[2,3]$.

We present a case of a synchronous diagnosis of cSCC and Waldenström's macroglobulinemia with an associated lung mass. With the pulmonary histology confirming squamous differentiation, the considered origin of the lung mass was either a primary SCC of the lung or metastatic cSCC. To our knowledge, this is the first case report detailing these coexisting diagnoses. The report highlights difficulties in diagnosis and management, particularly in a patient with multiple synchronous malignancies. It further emphasizes the need for expanded availability of specific therapies, including PD-1 inhibitors, in Africa.

\section{Case Presentation}

\section{Clinical Presentation and Diagnosis}

A 61-year-old male smoker with a 55-pack-year smoking history was referred with an ulcerating wound on the dorsum of the right hand, with poor wound healing and an associated persistent leukocytosis. On presentation, the patient described generalized myalgia but gave no history of constitutional symptoms, no symptoms related to hyperviscosity and had no features to suggest sepsis. The skin lesions had progressed rapidly within the preceding 2 weeks since a visit to his general practitioner and had coalesced into a single large lesion.

Despite a history of a recent lion bite to the other hand, the concern for sepsis and bacteremia appeared invalid considering the absence of temperature spikes and repeatedly normal blood cultures with no growth demonstrated. However, the patient did have a leukocyte count of $43 \times 10^{9} / \mathrm{L}$ with a marked monocytosis $\left(2.69 \times 10^{9} / \mathrm{L}\right.$ or $\left.6.2 \%\right)$ and a neutrophilia $\left(36.86 \times 10^{9} / \mathrm{L}\right.$ or $\left.84.9 \%\right)$, a C-reactive protein level of $52.3 \mathrm{mg} / \mathrm{L}$ and a procalcitonin level of $0.34 \mathrm{ng} / \mathrm{mL}$. He had an associated normochromic normocytic anemia (hemoglobin $11.3 \mathrm{~g} / \mathrm{dL}$, MCV $96.0 \mathrm{fL}$ and MCHC $33.2 \mathrm{~g} / \mathrm{dL}$ ) with rouleaux formation but was normocalcemic at 2.33 $\mathrm{mmol} / \mathrm{L}$ adjusted calcium.

The persistence of the leukocytosis in the setting of mild hepatosplenomegaly detected on abdominal ultrasound, at 18 and $13 \mathrm{~cm}$, respectively, prompted an investigation with bone marrow aspiration and trephine biopsy. The trephine biopsy confirmed the presence of a lymphoplasmacytic infiltrate estimated at 30\% within the marrow but with maintained hemopoietic reserve (Fig. 1A-D). Immunophenotypic analysis revealed 3\% small clonal B-cells which expressed $\mathrm{Kappa}^{++}, \mathrm{CD} 19^{+/++}, \mathrm{CD} 20^{++/++}, \mathrm{CD} \mathrm{bb}^{++}$, and CD22 $2^{+}$. The cells did not express CD5, CD10, or CD43. Serum protein electrophoresis demonstrated an "M" component of $24 \mathrm{~g} / \mathrm{L}$ with a severe immunoparesis with the band typing as IgM kappa (serum IgM 34.1 $\mathrm{g} / \mathrm{L}$ ). Testing for the MYD88 L265P mutation was performed but the mutation was not detected. Despite the absence of the MYD88 L265P mutation, a diagnosis of Waldenström's macroglobulinemia was made on the basis of a lymphoplasmacytic lymphoma on trephine biopsy, in the setting of the IgM monoclonal gammopathy.

Owing to the size and rapidity of progression of the skin lesions they were excised by a plastic surgeon and reconstructed with full-thickness grafts from the lower abdomen. Intra-

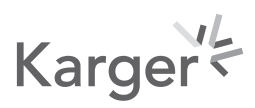




\section{Case Reports in Oncology}

Case Rep Oncol 2020;13:1474-1482

\begin{tabular}{l|l}
\hline DOI: 10.1159/000511460 & ( 2020 The Author(s). Published by S. Karger AG, Basel
\end{tabular} www.karger.com/cro

Limberis et al:: A Case of Synchronous Waldenström's Macroglobulinemia and cSCC with a Lung Mass
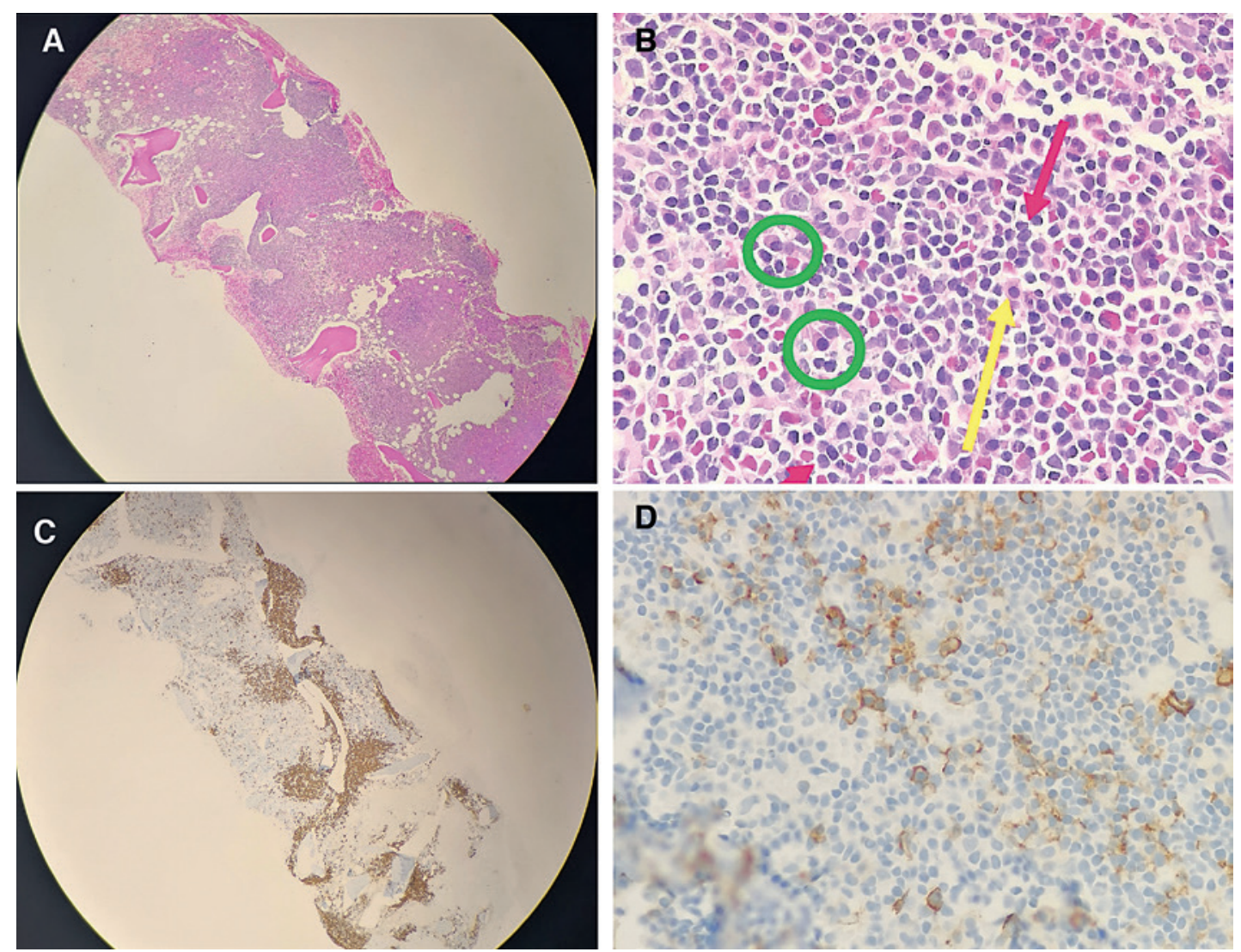

Fig. 1. Bone marrow trephine biopsy. A Bone marrow trephine at $\times 4$ magnification, showing hypercellular bone marrow and hyperchromatic areas of nodular and paratrabecular infiltrates. B Bone marrow trephine at $\times 50$ magnification. The hyperchromatic areas comprise small lymphoid cells with mature appearance (red arrows), admixed plasma cells (green circles) and associated mast cells (yellow arrow). C Bone marrow trephine immunohistochemistry. CD20 highlights the B-lineage infiltrate with the paratrabecular and nodular infiltrate comprising about $30 \%$ of the marrow surface area. D Bone marrow trephine Immunohistochemistry. CD138 staining highlights the admixed plasma cells.

operative pathological findings confirmed that the lesions were clear at the deep margins, allowing the preservation of extensor tendons and other related structures.

Histology from lesions on the dorsum of the hand, second webspace, and dorsum of the thumb were compatible with an invasive well-differentiated keratinizing SCC (Fig. 2A). A p16INKa stain showed a positive block pattern of staining (Fig. 2B). Considering the rapidity of progression and the synchronous cancer diagnosis, a workup for immunocompromise was conducted, including the addition of assessment for human papillomavirus (HPV). PCR for HPV was performed on two occasions and confirmed the presence of HPV 16 and 45, utilizing the QIAGEN QIAMP DNA FFPE tissue Kit.

A full malignant workup further revealed a right apical pulmonary mass, with associated erosion of the third posterior rib and a suspicious right lower lobe nodule (Fig. 3A, B). There were no additional lytic bony lesions; however, positron emission tomography-computed tomography (F-18 FDG PET/CT) confirmed a SUV $\mathrm{max}_{\text {in }}$ in the apical mass of 3.76, and further demonstrated diffuse radiotracer uptake throughout the appendicular and axial skeleton with the highest $\mathrm{SUV}_{\max }$ of 4.81 (Fig. 3C, D). These findings were attributed to bone marrow involvement by the Waldenström's macroglobulinemia. 


\section{Case Reports in Oncology}

Limberis et al.: A Case of Synchronous Waldenström's Macroglobulinemia and cSCC with a Lung Mass
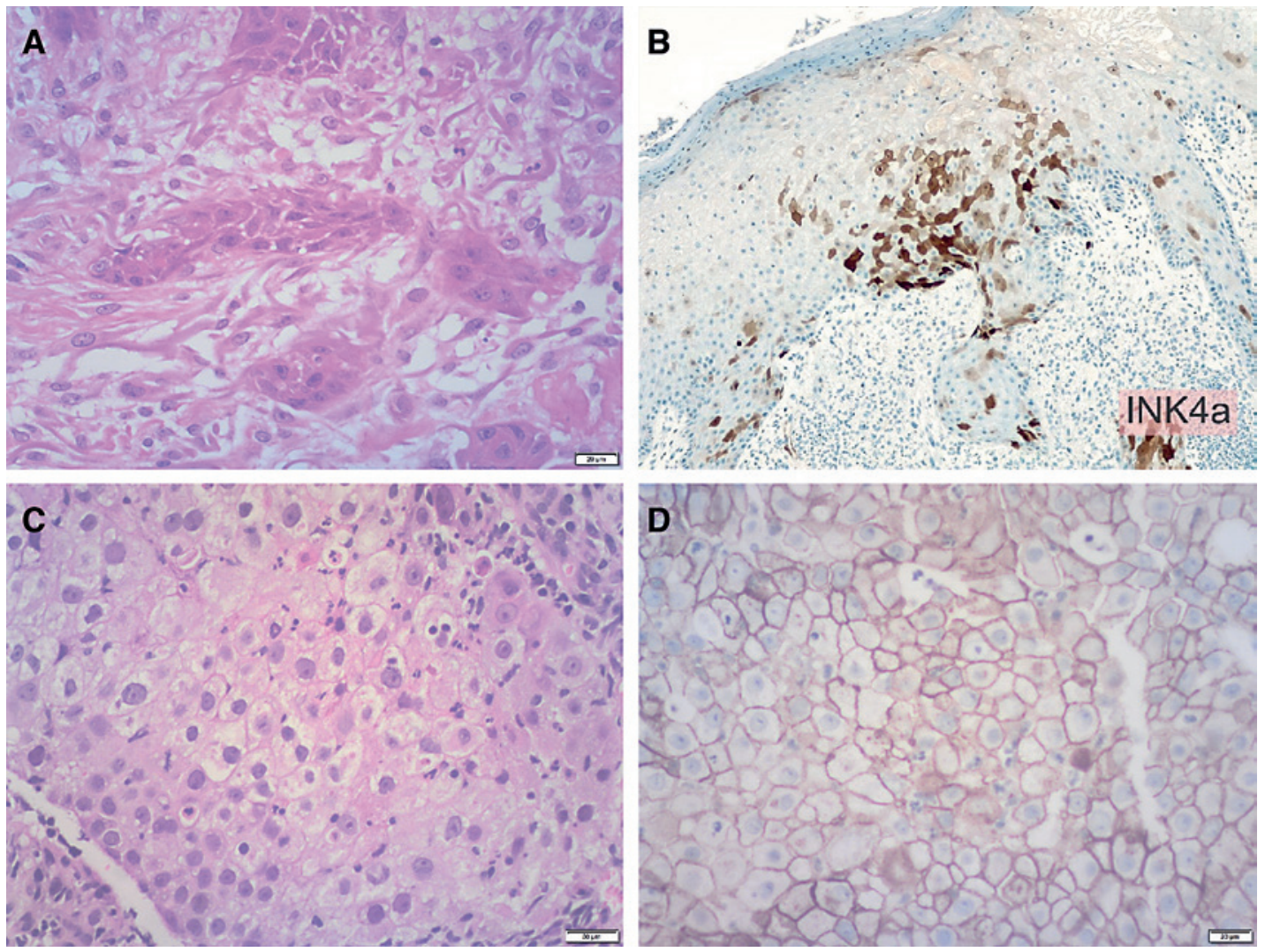

Fig. 2. Histopathology. A High-power view of the skin demonstrating an invasive well-differentiated keratinizing squamous cell carcinoma. B p16INKa stain showed a positive block pattern of staining. C Histology of the apical lung mass confirmed a moderately differentiated squamous cell carcinoma, with some areas suggestive of glandular differentiation and some areas of clear cell vacuolated cytoplasm. D PD-L1 was strongly positive in more than $95 \%$ of tumor cells (TPS >95\%).

The pulmonary mass was biopsied under CT guidance. The core biopsies performed on the apical lung mass confirmed a moderately differentiated SCC, with some areas suggestive of glandular differentiation and some areas of clear cell vacuolated cytoplasm (Fig. 2C). Stains confirming squamous differentiation, CK5/6, and p63, were positive, while Napsin-A and thyroid transcription factor-1 (TTF-1) were negative. PCR showed HPV 6 to be present. PD-L1 was strongly positive in more than 95\% of tumor cells (TPS $>95 \%$ ) (Fig. 2D).

\section{Treatment and Outcome}

Diagnosis and management of this patient proved to be a challenge, owing to the complexity of the case and the synchronous malignant disease diagnosed. The origin of the lung mass was difficult to determine with a third primary malignancy in the form of lung cancer being a possibility, particularly considering the significant smoking history, but with an inability to exclude a metastatic cSCC entirely.

Considering the rapid progression of the cutaneous lesions, the primary therapy initiated in our case was the full excision of the cutaneous lesions, with histological sampling done simultaneously. Despite the initial absence of constitutional symptoms, the patient subsequently developed progressive loss of weight and worsening pain in the chest secondary to 


\section{Case Reports in Oncology}

Case Rep Oncol 2020;13:1474-1482

\begin{tabular}{l|l}
\hline DOI: $10.1159 / 000511460$ & (c) 2020 The Author(s). Published by S. Karger AG, Basel
\end{tabular} www.karger.com/cro

Limberis et al.: A Case of Synchronous Waldenström's Macroglobulinemia and cSCC with a Lung Mass
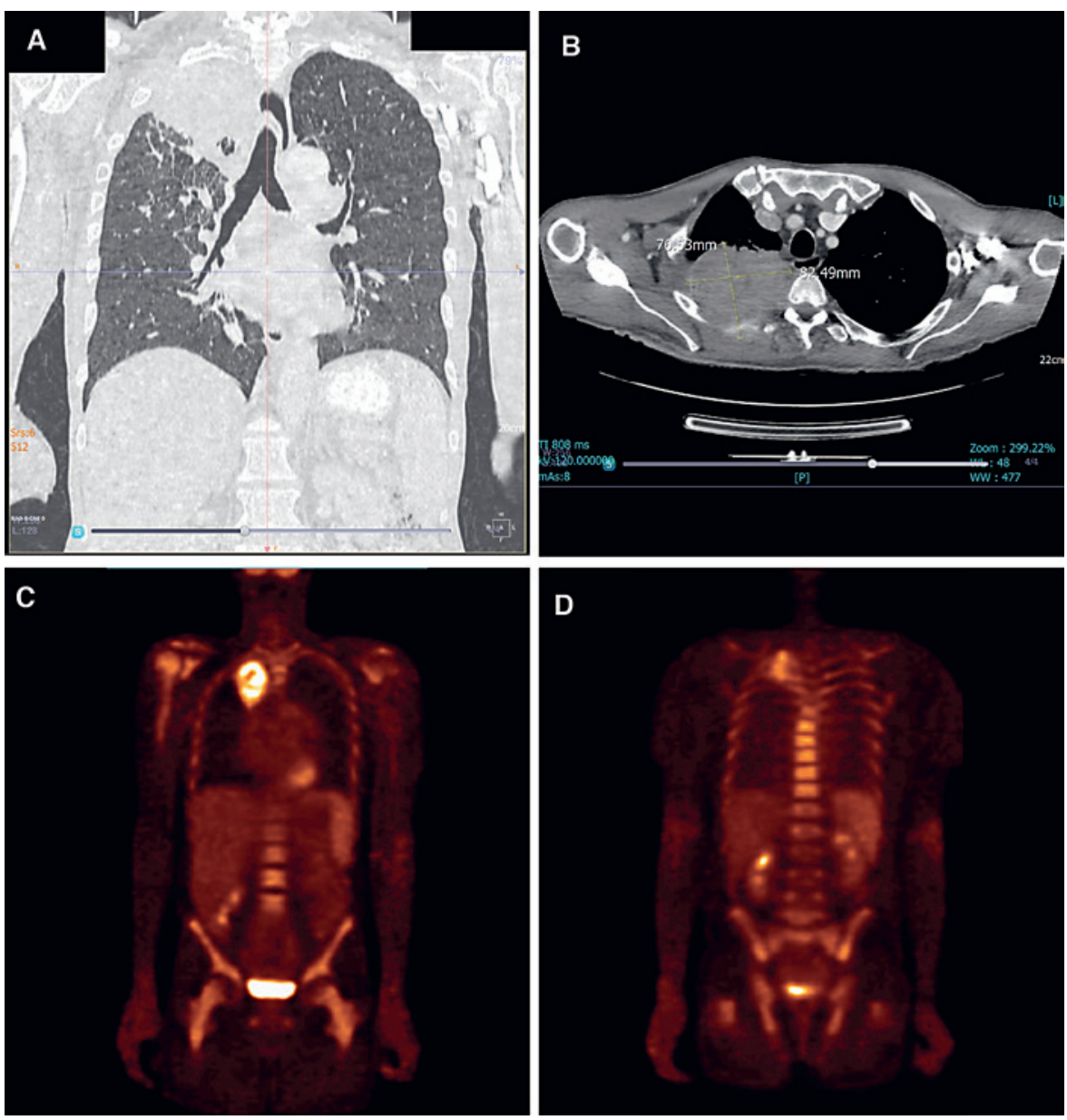

Fig. 3. Imaging. A Computed tomography chest scan - coronal view, demonstrating a large right apical lung mass and right lower lobe nodule. B Computed tomography chest scan - axial view, demonstrating a large apical lung mass with posterior chest wall infiltration and erosion of the third rib. C, D F-18 FDG PET/CT coronal views, demonstrating diffuse radiotracer uptake throughout the appendicular and axial skeleton (with the highest $\mathrm{SUV}_{\max }$ of 4.81) and a metabolically active apical lung mass $\left(\operatorname{SUV}_{\max } 3.76\right)$.

the pulmonary lesion with associated bone involvement. He had no additional symptoms that could be attributed to the Waldenström's macroglobulinemia alone, so he was managed with a primary focus on the lung mass. At the time of initiation of therapy, he had an Eastern Cooperative Oncology Group (ECOG) performance status of 1.

Taking into account the strong PD-L1 positivity, we considered the use of pembrolizumab as either first- or second-line therapy in our patient, but it was not funded in this case [4]. Cemiplimab was also considered but was unavailable on the expanded access program [5]. In the absence of immunotherapy options, we opted for a regimen that had demonstrated potential efficacy against both advanced primary lung cancer and metastatic cSCC. A platinum-based chemotherapy regimen in the form of carboplatin and paclitaxel was initiated. The follow-up imaging done after three cycles of chemotherapy showed disease progression, both in the apical pulmonary mass and the right lower lobe mass. In addition, there was a prominent paratracheal $4 \mathrm{R}$ lymph node of $11 \mathrm{~mm}$ noted. The patient was planned for secondline therapy, but due to severe pain, he was sent for palliative radiotherapy to the right upper lobe of the lung. He started radiotherapy at 1.8 Gy per day for 5 days, but due to deterioration 
Limberis et al.: A Case of Synchronous Waldenström's Macroglobulinemia and cSCC with a Lung Mass

in his condition he was converted to a more palliative course with larger fractions at 3 Gy daily for 4 days. Shortly after the completion of the radiotherapy, the patient demised, about 6 months after the initial presentation.

\section{Discussion}

Non-melanoma skin cancer poses an increasing burden on health systems worldwide. cSCC is second only to basal cell carcinoma as the most common cutaneous malignancy. While the burden of advanced cSCC is small, it carries with it a significant risk of metastases and death [1]. Risk factors for advanced disease and metastasis were present in our patient, including a depth of tumor invasion of more than $2 \mathrm{~mm}$, anatomic location on the hands, multiple tumors and immunosuppression [6].

A Waldenström's macroglobulinemia was diagnosed concurrently in our patient, which can account for immunosuppressive risk. Several studies have suggested an increase in malignancies in both patients with Waldenström's macroglobulinemia and their kin, and have drawn inferences on cancer clustering amongst these patients [2]. A large study analyzing SEER data has shown an increased incidence of both solid tumors (including lung cancer) and hematological malignancies in patients with Waldenström's macroglobulinemia, versus the general population [3]. There is, however, little evidence describing an association between cSCC and Waldenström's macroglobulinemia. Hanzis et al. [2] found that of their 225 patients and kin with an associated malignancy, 7.1\% had a non-melanoma skin cancer, though the bulk of these diagnoses predated the diagnosis of the Waldenström's macroglobulinemia.

Immunohistochemistry and PCR analysis performed on the skin biopsy of our patient confirmed the presence of human papillomavirus, specifically recording HPV serotypes 16 and 45. Recent prospective data has demonstrated the role of HPV in carcinogenesis of head and neck cancers [7]. HPV oncogenesis in cSCC has been described in more recent years, with its role appearing to be that of a co-factor to ultraviolet-induced DNA mutations. The beta HPV types have been more commonly implicated [8]. A 2019 study recently linked alpha HPV serotypes, most particularly serotype 16, to CSCC [9]. With all types of HPV being known to be more common in patients with immunocompromise, it is conceivable that it was found in our patient with an underlying Waldenström's macroglobulinemia [10].

The lung mass provided a diagnostic challenge. Histology demonstrated a SCC, but further investigation was unable to differentiate between a primary SCC of the lung and metastatic cSCC. The differing HPV serotypes between the skin lesions and the lung mass may point to a third primary malignancy in the lung, particularly in a patient with a significant smoking history, but there were no other features to confirm this.

Aside from the multiple synchronous malignancies, our patient was noted to be atypical in several other aspects. He was found to be MYD88 L265P mutation-negative. A Waldenström's macroglobulinemia is diagnosed with a lymphoplasmocytic infiltrate in the bone marrow and the detection of a monoclonal IgM gammopathy with the mutation not essential for diagnosis, despite it being present in the majority of patients. About $5-10 \%$ of patients with Waldenström's macroglobulinemia have been shown to have no expression of MYD88 L265P [11]. He was classified as a low-risk patient according to the International Prognostic Scoring System for Waldenström's macroglobulinemia; however, the presence of constitutional symptoms may suggest advanced disease and a poor outcome [12]. A further challenge arises in that the constitutional symptoms in our case could have been attributed to either the cSCC (mainly if it was indeed metastatic), possible lung cancer, or Waldenström's macroglobulinemia.

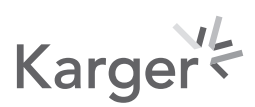


Furthermore, the presence of diffuse radiotracer uptake on the F-18 FDG PET/CT scan may be attributed to Waldenström's macroglobulinemia, as it is demonstrated that about $40 \%$ of patients with the disease have an FDG PET-positive bone marrow signal; however, the role of PET scanning in Waldenström's macroglobulinemia is poorly defined [13]. Data regarding the use of F-18 FDG PET/CT in CSCC is also scanty; however, it has been noted that sensitivity is high for detecting both primary and secondary cSCC lesions [14]. The intense FDG avidity demonstrated in the right apical lung mass in our patient is in keeping with the malignant process confirmed on biopsy; however, whether this represents metastatic cSCC or a new primary cancer is still unclear.

The irregularities and complexities of the case made management a challenge. We elected to adopt a watch-and-wait strategy regarding Waldenström's macroglobulinemia and to manage with a primary focus on the lung mass.

Metastatic CSCC is managed typically with medical therapy given with palliative intent, but reliable and supportive data for systemic therapy is scanty. Historically, cytotoxic chemotherapy regimens have been used as the conventional treatment modality, with platinum-based treatments being used most commonly. The considered agents include platinum therapies (both cisplatin and carboplatin), taxanes, 5-fluorouracil, capecitabine, bleomycin, interferon-alpha, doxorubicin, and ifosfamide. However, none of these agents given singly or in combination has been approved for standard use on the basis of inconclusive evidence [6].

The latest breakthrough in treatment, however, has been the 2020 approval by the FDA of pembrolizumab for patients with recurrent or metastatic CSCC who are not candidates for curative surgery or radiation. The approval was granted on the outcome of the phase II KEYNOTE-629 study which showed a response rate of 34.3\% (95\% CI 25.3-44.2) and a disease control rate of $52.4 \%$ (95\% CI 42.4-62.2) with a median response duration not reached (range 2.7-13.1+ months) [4]. This follows on the back of the 2018 FDA approval, and the 2019 EMA conditional approval, of cemiplimab for patients with metastatic or locally advanced cSCC who are not candidates for curative surgery or radiation. The decision has been based on a trial published in the New England Journal of Medicine (NEJM) that demonstrated a response rate of $47 \%$ (95\% CI 35-59) in the 75 patients with metastatic disease in the phase I and II trials. Therapy was generally well tolerated, with about $7 \%$ of patients discontinuing treatment owing to adverse effects [5]. Of mention regarding our patient, there has been little consensus on the use of PD- 1 inhibitors in immunocompromised patients $[15,16]$.

Advanced SCC of the lung is generally managed with platinum-based doublets as firstline therapy, though treatment with pembrolizumab in combination with standard chemotherapy, including carboplatin and paclitaxel, did result in prolonged overall survival in metastatic patients $[17,18]$.

Our patient was treated with a platinum-based regimen of carboplatin and paclitaxel, which was funded by the patient's medical aid. Other therapeutic options were not available in this case owing to both affordability and limited availability. We question whether the outcome would have been different should we have had access to a broader range of therapies.

To our knowledge, we provide one of the first accounts, both in Africa and worldwide, of a synchronous diagnosis of Waldenström's macroglobulinemia, cSCC, and a lung mass with squamous differentiation. The case highlights the potential difficulties in diagnosis and symptom attribution in patients with multiple concurrent malignancies. It further highlights the need for wider global access to targeted therapies and immune checkpoint inhibitors. 


\section{Case Reports in Oncology}

\begin{tabular}{l|l}
\hline Case Rep Oncol 2020;13:1474-1482 \\
\hline DOI: 10.1159/000511460 & $\begin{array}{l}\text { @ 2020 The Author(s). Published by S. Karger AG, Basel } \\
\text { www.karger.com/cro }\end{array}$ \\
\hline
\end{tabular}

Limberis et al.: A Case of Synchronous Waldenström's Macroglobulinemia and cSCC with a Lung Mass

\section{Acknowledgments}

We acknowledge the following doctors who made contributions to the manuscript in their relevant specialties: Dr. Antonet de la Rey, Consultant Radiologist; Dr. Karissa Mannaru, Consultant Hematopathologist; Dr. Janet Reed, Nuclear Medicine Registrar.

\section{Statement of Ethics}

Prior to his demise, written informed consent for the publication of his case was obtained from the patient.

\section{Conflict of Interest Statement}

The authors have no conflicts of interest to declare.

\section{Funding Sources}

There was no funding for this publication.

\section{Author Contributions}

C.L. Limberis and B.L. Rapoport treated the patient and conceptualized the manuscript. C.L. Limberis drafted the manuscript. S. Nayler drafted sections of the manuscript pertaining to histopathology. B.L. Rapoport reviewed and edited the manuscript. All authors have read and approved the final version of the manuscript.

\section{References}

1 Schmults CD, Karia PS, Carter JB, Han J, Qureshi AA. Factors predictive of recurrence and death from cutaneous squamous cell carcinoma: a 10-year, single-institution cohort study. JAMA Dermatol. 2013;149(5):541-7.

2 Hanzis C, Ojha RP, Hunter Z, Manning R, Lewicki M, Brodsky P, et al. Associated malignancies in patients with Waldenström's macroglobulinemia and their kin. Clin Lymphoma Myeloma Leuk. 2011;11(1):88-92.

3 Castillo JJ, Olszewski AJ, Hunter ZR, Kanan S, Meid K, Treon SP. Incidence of secondary malignancies among patients with Waldenström macroglobulinemia: An analysis of the SEER database. Cancer. 2015;121(13): 2230-6.

4 Grob JJ, Gonzalez R, Basset-Seguin N, Vornicova O, Schachter J, Joshi A, et al. Pembrolizumab Monotherapy for Recurrent or Metastatic Cutaneous Squamous Cell Carcinoma: A Single-Arm Phase II Trial (KEYNOTE-629) [published online ahead of print, 2020 Jul 16]. J Clin Oncol. 2020;JC01903054.

5 Migden MR, Rischin D, Schmults CD, Guminski A, Hauschild A, Lewis KD, et al. PD-1 Blockade with Cemiplimab in Advanced Cutaneous Squamous-Cell Carcinoma. N Engl J Med. 2018;379(4):341-51.

6 Burton KA, Ashack KA, Khachemoune A. Cutaneous Squamous Cell Carcinoma: A Review of High-Risk and Metastatic Disease. Am J Clin Dermatol. 2016;17(5):491-508.

7 Agalliu I, Gapstur S, Chen Z, Wang T, Anderson RL, Teras L, et al. Associations of Oral $\alpha-$, $\beta$-, and $\gamma$-Human Papillomavirus Types With Risk of Incident Head and Neck Cancer. JAMA Oncol. 2016;2(5):599-606.

8 Rollison DE, Viarisio D, Amorrortu RP, Gheit T, Tommasino M. An Emerging Issue in Oncogenic Virology: the Role of Beta Human Papillomavirus Types in the Development of Cutaneous Squamous Cell Carcinoma. J Virol. 2019;93(7):e01003-18.

9 Aoki R, Clanner-Engelshofen BM, Charnowski S, Ruzicka T, Reinholz M. Distribution of high-risk $\alpha$-genus human papillomavirus genotypes impacts cutaneous neoplasms. J Eur Acad Dermatol Venereol. 2019;33(7): 1304-11.

10 Burd EM, Dean CL. Human Papillomavirus. Microbiol Spectr. 2016;4(4).

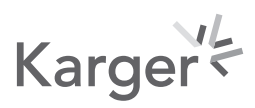


11 Treon SP, Xu L, Yang G, Zhou Y, Liu X, Cao Y, et al. MYD88 L265P somatic mutation in Waldenström's macroglobulinemia. N Engl J Med. 2012;367(9):826-33.

12 Morel P, Duhamel A, Gobbi P, Dimopoulos MA, Dhodapkar MV, McCoy J, et al. International prognostic scoring system for Waldenstrom macroglobulinemia. Blood. 2009;113(18):4163-70.

13 Banwait R, O'Regan K, Campigotto F, Harris B, Yarar D, Bagshaw M, et al. The role of 18F-FDG PET/CT imaging in Waldenstrom macroglobulinemia. Am J Hematol. 2011;86(7):567-72.

14 Mahajan S, Barker CA, Singh B, Pandit-Taskar N. Clinical value of 18F-FDG-PET/CT in staging cutaneous squamous cell carcinoma. Nucl Med Commun. 2019;40(7):744-51.

15 Pianko MJ, Moskowitz AJ, Lesokhin AM. Immunotherapy of Lymphoma and Myeloma: Facts and Hopes. Clin Cancer Res. 2018;24(5):1002-10.

16 Fisher J, Zeitouni N, Fan W, Samie FH. Immune checkpoint inhibitor therapy in solid organ transplant recipients: A patient-centered systematic review. J Am Acad Dermatol. 2020;82(6):1490-500.

17 Planchard D, Popat S, Kerr K, Novello S, Smit EF, Faivre-Finn C, et al. Metastatic non-small cell lung cancer: ESMO Clinical Practice Guidelines for diagnosis, treatment and follow-up [published correction appears in Ann Oncol. 2019 May;30(5):863-870]. Ann Oncol. 2018;29(Suppl 4):iv192-iv237.

18 Paz-Ares L, Luft A, Vicente D, Tafreshi A, Gümüș M, Mazières J, et al. Pembrolizumab plus Chemotherapy for Squamous Non-Small-Cell Lung Cancer. N Engl J Med. 2018;379(21):2040-51. 